\title{
Navigational Consistency in Websites: What Does it Mean to Users?
}

\author{
Helen Petrie, George Papadofragkakis, Christopher Power, and David Swallow \\ Department of Computer Science, University of York, Heslington, \\ York YO10 5DD, United Kingdon \\ \{helen.petrie, gp519, cpower, dswallow\}@cs.york.ac.uk
}

\begin{abstract}
A study was conducted to investigate the effects of navigational inconsistencies in websites on users' perceptions and performance. Of four inconsistencies manipulated (position of navigational bar, order of elements in navigational bar, font type and size of elements), only the position of the bar had a substantial effect. However this affected both users' perception of their performance and their actual performance, in terms of the time spent on webpages. The mean time spent on the page with the inconsistently positioned navigational bar more than doubled and this effect persisted over subsequent pages. The methodology developed provides a useful way of investigating the effects of navigational inconsistency, an under-studied phenomenon.
\end{abstract}

Keywords: Website navigation, navigational consistency, website usability.

\section{Introduction}

Ever since we have had hypertext systems, people have been getting lost in them. The term "lost in hyperspace" was coined in the late 1980s [2] to describe this phenomenon, even before the advent of the World Wide Web. Many navigational aids have been proposed to users to assist in overcoming this problem, including breadcrumb trails [6, 13], site maps [1, 13] and tabs [13]. Nonetheless, users continue to get lost and feel disoriented, particularly on the Web.

In addition, numerous guidelines and design principles have been proposed to assist developers in creating websites that are easy to navigate [3, 4]. One such guideline is to use consistent navigation, which is of course simply a specific instantiation of the more general and widely cited principle of consistency in interface design [8, $10,12]$. But what do users actually notice in navigational consistency and what affects their performance? Surprisingly few empirical studies have been conducted on these questions. Studies have investigated the sense of "lostness" at a global level and created appropriate metrics $[5,9,11]$ with interesting results, but less work has looked at the fine-grained detail of navigational consistency. Yet the few studies which have done so have produced surprising results. For example, Kalbach and Bosenick [7] found that users did not perform any more efficiently when the navigation was on the lefthand side of webpages, as recommended [13], than when they were on the righthand side of pages. 
This study therefore set out to investigate both user perceptions and performance in relation to four aspects of navigational consistency on websites: position of the navigational bar on the webpage, order of elements in a navigational bar, size and font of navigational elements.

\section{Method}

Fourteen participants, nine men and five women were recruited from students at the University of York. Their mean age was 20.9 years (range: 19 to 25). They were all experienced Web users, with a mean of 8.3 years of experience (range: 3 to 11). They spent on average 35.1 hours a week using the Web (range: 10 to 70).

We have developed a website specifically to enable us conduct studies of navigation on websites. It is the website for a fictional university computer science department, the University of North Yorkshire. The website contains information about four MSc courses, each of which had one distinct subtree within the website's information architecture with no cross-linking between them. This means that when participants undertake a task about one MSc, a navigational inconsistency can be introduced and it is highly likely they will all encounter it after the same number of pages and will then continue to the task conclusion with the same number of pages with the new navigational style (data from participants who deviate from the expected path can be removed from analyses if necessary).

The navigational inconsistencies investigated in this study were:

- Position of navigational bar: changed from lefthand to righthand side of page;

- Order of elements in the navigational bar: the order of the 5 elements was altered;

- Font size: changed from 10 pt to $12 \mathrm{pt}$;

- $\quad$ Font type: changed from sans serif (Arial) to serif (Times Roman).

10 and 12 point were chosen for font sizes, as although the difference in size is not great, an informal survey of 100 websites showed that these are the two most commonly used default font sizes. Although much is presented in Times Roman, there is a long running discussion about the readability of serif versus sans serif fonts, and this distinction is quite clear to readers, so a serif and a sans serif font were used.

A Windows XP Professional PC was used to conduct the study. Screen resolution was set at 1024 x 768, and no horizontal scrolling was necessary in accessing the study website. Participants were given the choice between using Internet Explorer 6.0.2900 and Mozilla Firefox 3.0.8, as it was known that the student population at York favour these two browsers. Thus any difficulties in navigation would not be attributable to lack of familiarity with the browser. Four chose Explorer and 10 chose Firefox. There was no difference in the website's appearance between the two browser configurations, so this choice did not affect the final results. Morae software ${ }^{1}$ was used to record participants' interaction with the website.

\footnotetext{
${ }^{1}$ Techsmith. Morae customer experience software. Available at: www.techsmith.com/ morae.asp
} 
Participants undertook ten tasks, eight of which led the participant through a sequence of pages that introduced one navigational inconsistency. The remaining two tasks were control tasks and they led participants through a sequence of pages with no inconsistency, that is a consistent sequence of pages.

After each task the participant answered the following question: Did you notice any change in the navigation bars throughout this task? (answer yes or no). If they answered yes, they were asked two further questions: What was/were the change(s)? Did the change(s) affect your performance during the task? (on a scale from $1=$ not at all to $5=$ very much)

No feedback was given to the participant about the navigational (in)consistencies and the accuracy of their perceptions after each task. After all the tasks were completed, the participants were debriefed and were shown the inconsistencies.

\section{Results}

The extent to which participant noticed the navigational inconsistencies was analysed. The change in the position of the navigational bar was noticed on most occasions $(85.7 \%)$, the change in font size of navigational elements was noticed on about half the occasions (42.9\%) and the change in font type and the order of navigational elements was noticed on only about $20 \%$ of instances $(21.4 \%$ in both cases). A Cochran's Q test showed that these percentages were significantly different from each other (test conducted on mean $\%$ per participant per inconsistency type, Cochran's $\mathrm{Q}=44.1, \mathrm{df}=3, \mathrm{p}<0.000$ ).

For those participants who noticed a navigational inconsistency, their perception of how it affected them was analysed, using their answers on the Lickert scale $(1=$ not at all to $5=$ very much). The mean rating for the perception of the position inconsistency was 2.38 , for the order and font type inconsistency it was 1.67 and for the font size inconsistency it was 1.38 . Thus none of the mean ratings were higher than the midpoint on the rating scale.

To investigate how the inconsistencies affected participants' performance, the times they spent on the pages before the inconsistency (PagesB), the page where the inconsistency appeared (PageI) and the pages after the inconsistency (PagesA) were analysed. A one way repeated measures ANOVA was conducted on the mean time (in seconds) spent on the web pages with three variables: (1) the type of inconsistency, (2) the pages before/on/after the inconsistency, and (3) the task (first or second task for a particular inconsistency). This analysis found that there was a significant difference between the mean ratings for the five inconsistency conditions $(\mathrm{F}=5.86, \mathrm{df}=4$,

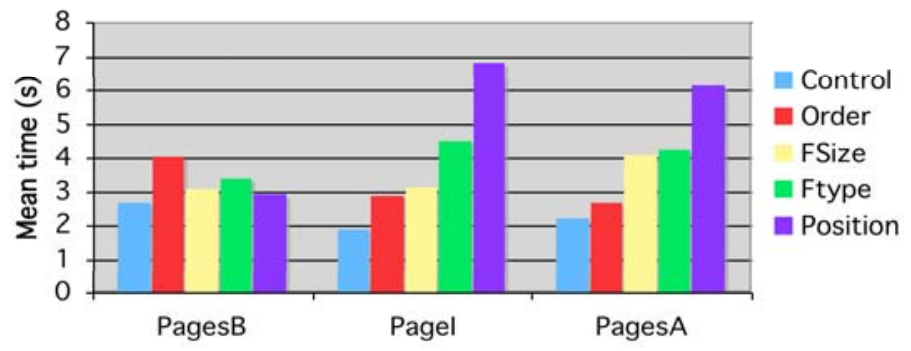

Fig. 1. Mean time on page for different navigational inconsistencies 
$52, \mathrm{p}<0.03)$. There was also a significant difference between the page conditions $(\mathrm{F}$ $=10.44, \mathrm{df}=2,26, \mathrm{p}<0.005)$. There was also a significant interaction between inconsistency type and page type $(\mathrm{F}=4.26, \mathrm{df}=8,104, \mathrm{p}<0.05)$. From Fig 1 , it can be seen that for the position inconsistency, the time spent on the page where the inconsistency occurs is much longer, and this increased time persists to subsequent pages.

\section{Discussion and Conclusions}

This study found that of the navigational inconsistencies investigated, only the position of the navigational bar had any noticeable influence, but that this affected both participants' perception and their performance. The effect on performance was very noticeable, with time spent on the page with the inconsistency more than doubling, and that effect persisting over subsequent pages. The persistence of the effect is interesting: it may be that once a position inconsistency occurs, web users become less confident about the layout of the navigation, and spend more time scanning the page when they land on this. We will follow up this possibility with a study of navigational inconsistencies that uses eye tracking to study the pattern of page scanning. We believe the methodology developed in this study will be useful for investigating the perception and performance implications of navigational inconsistencies, as well as the effectiveness of navigational aids such as breadcrumb trails. We are already undertaking a further study that investigates a larger range of navigational inconsistencies and will follow this up with an investigation of breadcrumb trails.

\section{References}

1. Billingsley, P.A.: Navigation through hierarchical menu structures: Does it help to have a map? In: Proc. Human Factors and Ergonomics Society Annual Meeting, pp. 103-107 (1982)

2. Edwards, Hardman: Lost in hyperspace: cognitive mapping and navigation in a hypertext environment. In: McAleese, R. (ed.) Hypertext: theory into practice. Intellect Books, Exeter (1989)

3. Farkas, D.K., Farkas, J.B.: Guidelines for designing web navigation. Technical Communication 47(3), 341-358 (2000)

4. Fleming, J.: Web navigation: designing the user experience. O'Reilly, Sebastopol (1998)

5. Gwizdka, J., Spence, I.: Implicit measures of lostness and success in web navigation. Interacting with Computers 19(3), 357-369 (2007)

6. Instone, K.: Location, path and attribute breadcrumbs. In: 3rd Annual Information Architecture Summit, Baltimore, March 16-17 (2002),

http: //instone.org/breadcrumbs

7. Kalbach, J., Bosenick, T.: Web page layout: a comparison between left- and right-justified site navigation menus. Journal of Digital Information 4(1) (2003), http://jodi.tamu.edu/Articles/v04/i01/Kalbach/

8. Nielsen, J.: Heuristic evaluation. In: Nielsen, J., Mack, R.L. (eds.) Usability Inspection Methods. John Wiley \& Sons, New York (1994)

9. Otter, M., Johnson, H.: Lost in hyperspace: metrics and mental models. Interacting with Computers 13(1), 1-40 (2000) 
10. Shneiderman, B.: Designing the user interface: Strategies for effective human-computer interaction, 3rd edn. Addison-Wesley Publishing, Reading (1998)

11. Smith, P.A.: Towards a practical measure of hypertext usability. Interacting with Computers 8(4), 365-381 (1996)

12. Tognazzini, B.: First principles of interaction design, http: / / www.asktog.com/basics/firstPrinciples.html

13. U.S. Department of Health and Human Services. Research-based web design and usability guidelines (2006), http: / /www. usability.gov/pdfs / guidelines.html\#1 